
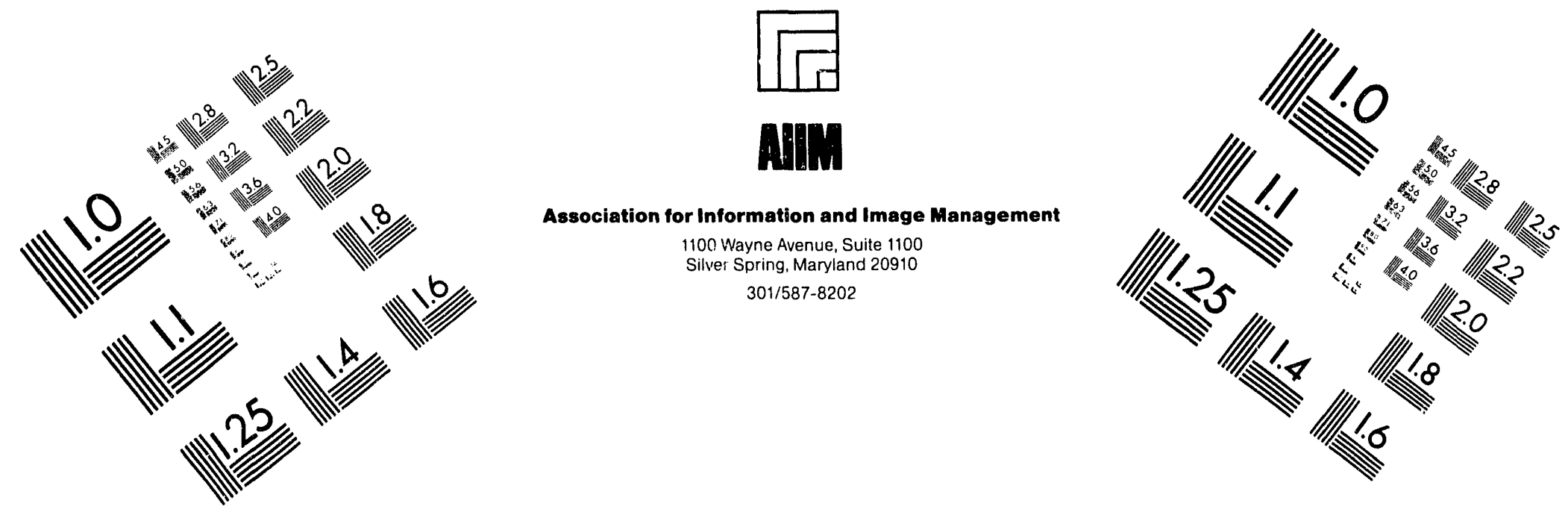

Centimeter

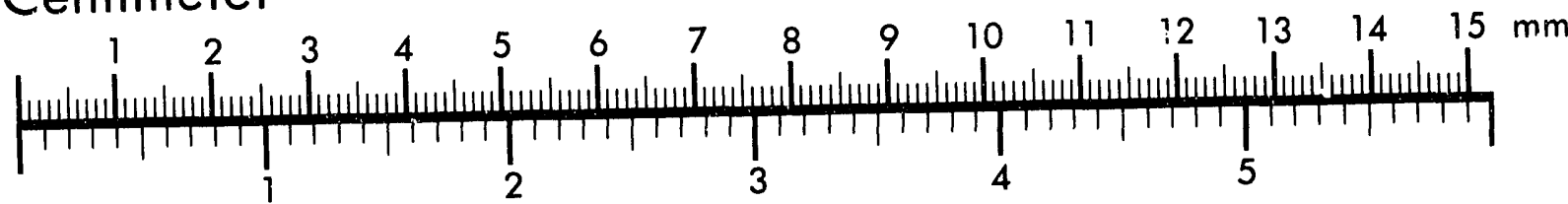
Inches
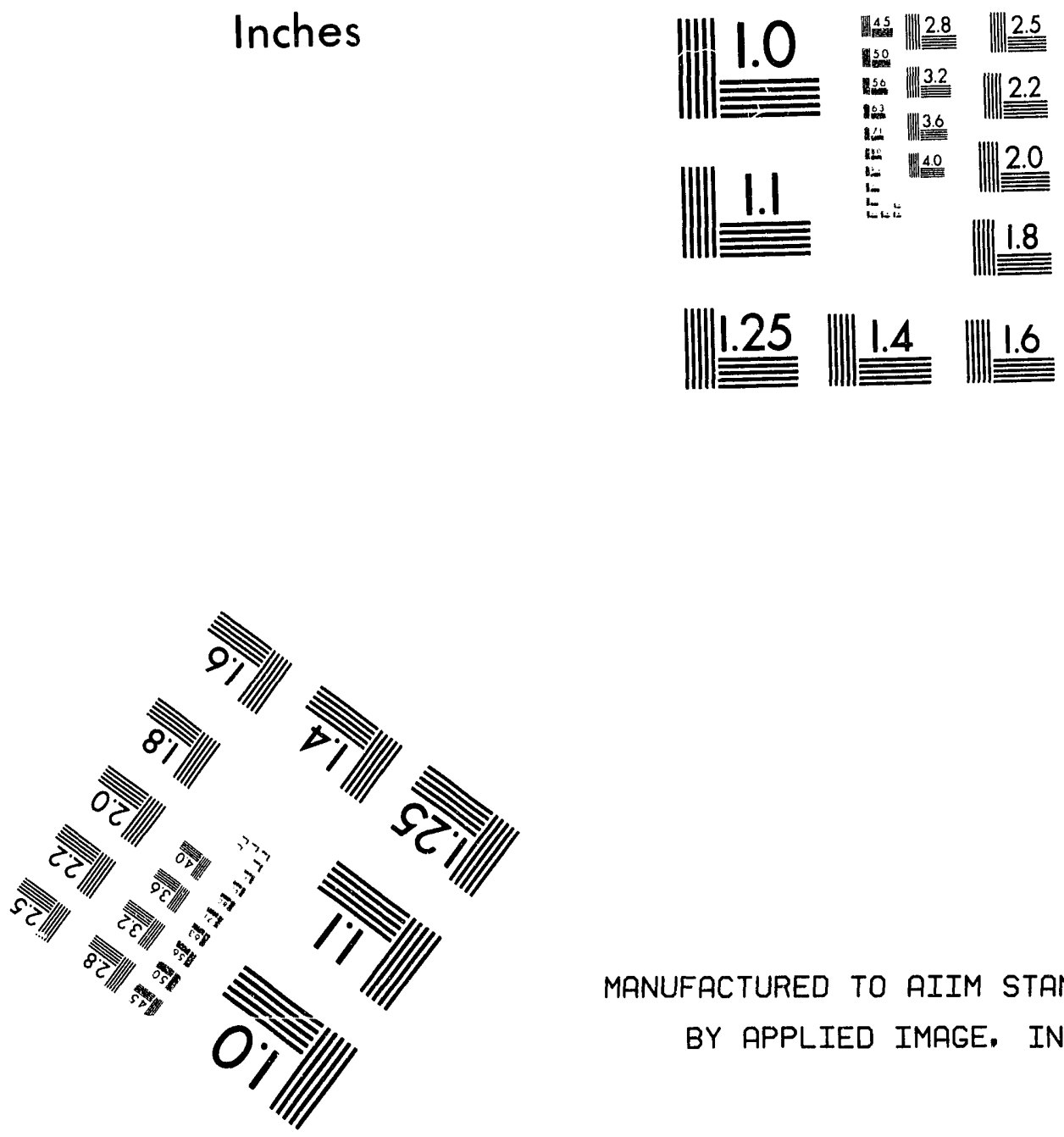

MANUFACTURED TO AIIM STANDARDS

BY APPLIED IMAGE. INC.

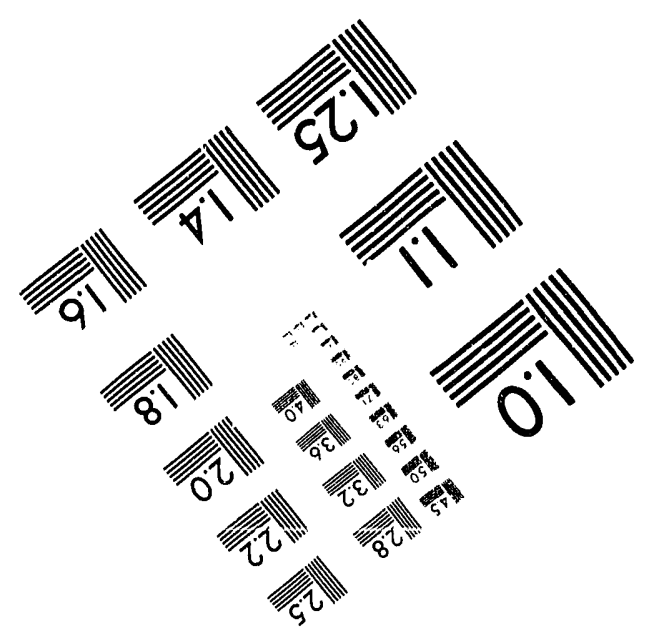



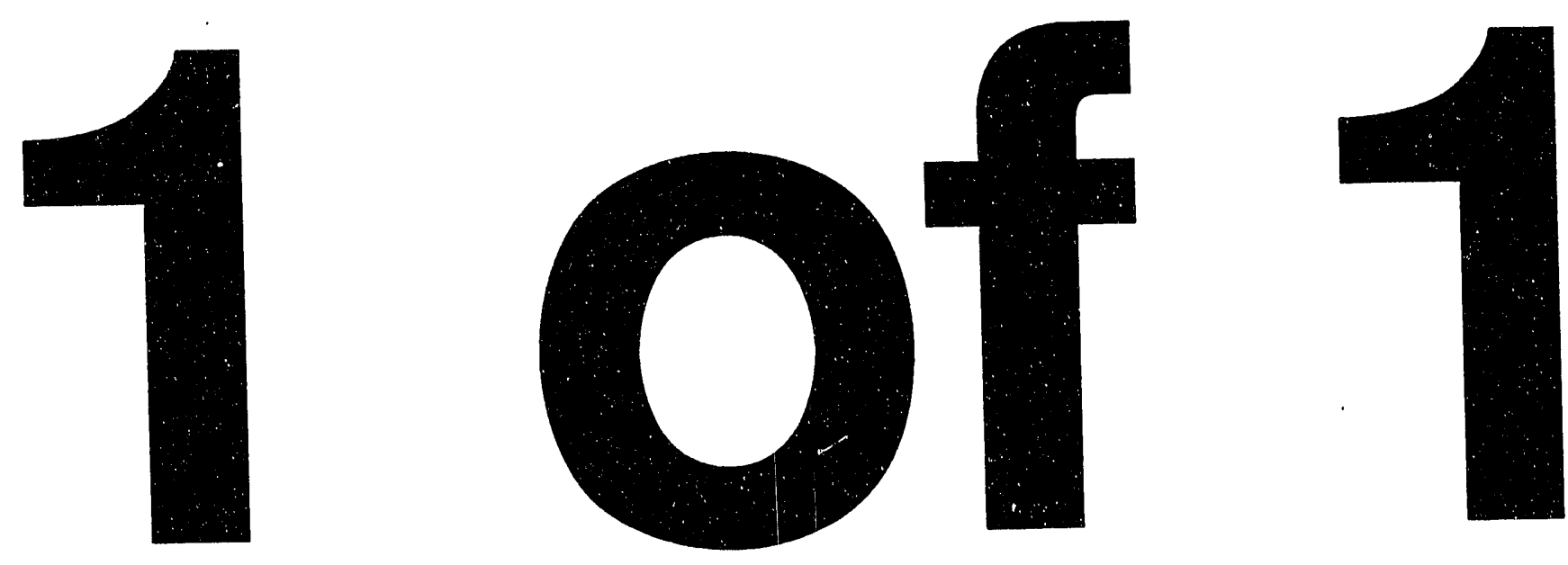


\title{
Optimal Magnet Sorting Procedure and Application to the APS Injector Synchrotron*
}

\author{
R. K. Koul, Fredrick Lopez and Fredrick E. Mills \\ APS, Argonne National Laboratory \\ Argonnc, IL 60439
}

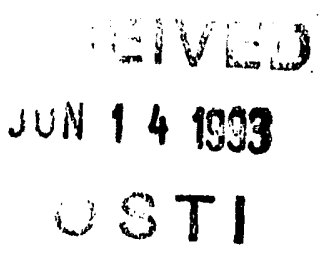

\section{Abstract}

A magnet sorting procedure for accelerators is developed. It is implemented in two steps. The first step is completely analytical in character while the second step involves the comparison of computed values with the measured crror values. The method has been implemented for sorting the Advanced Photon Source (APS) injector synchrotron dipoles and quadrupoles with excellent results.

\section{INTRODUCTION}

Suppose we are given a set of magnets whose magnetic field errors have been measured. Then the question arises whether there is some optimal way of placing them around the accelerator ring to minimize the effect of the magnetic field crrors.

One of the simplest ways to answer this question is to try all possible permutations of the known errors on the magnet locations and choose the combination which produces the minimum effect. However, the number of combinations grows as $n$ !, where $\mathrm{n}$ is the number of magnets to be sorted.

In the past, another method has been used for the simulated errors which, for lack of a better name, we will call the "Main Harmonic Cancellation Method" (see Lopez [1]). The idea of this method is as follows. Let us say we want to sort dipole magnets on dipole errors. The magnets of equal strength are selected and placed $\pi$ radians apart in phase of the particle trajectory so that the equal strength errors make equal but opposite contributions to the particle trajectory.

Sometimes the method of simulated annealing may be used for sorting. This method, also used in simulation for sorting the injector synchrotron dipoles over simulated crrors, gave a slight improvement over the Main Harmonic Cancellation Method when used in simulation for the APS injector synchrotron (see Sampson [2]). For details see Kirkpatrick [3] or Flannery [4].

\section{OPTIMAL PROCEDURE}

The new sorting procedure is based on solution of the linearized equations of motion. For details sec Koul [5] or Courant [6]. The solution characterizing the effect of errors is given by amplitude function I expressed below.

$$
I=C \sum_{i=1}^{i=n} \sum_{j=1}^{j=n} H\left(\beta_{i}, L_{i}\right) H\left(\beta_{j}, L_{j}\right) r_{i} f_{j} \cos \psi_{i j} .
$$

* Work supported by U.S. Department of Energy, Office of Basic Encrgy Sciences under Contract No. W-31-109-ENG-38.
$\mathrm{H}\left(\beta_{\mathrm{i}}, \mathrm{L}_{\mathrm{i}}\right)$ is some function of $\beta_{\mathrm{i}}$ and $\mathrm{L}_{\mathrm{i}}$, where $\beta_{\mathrm{i}}$ and $\mathrm{L}_{\mathrm{i}}$ are the beta function of the beam at the ith element and the length of the ith element respectively; $f_{\mathrm{i}}$ is the measured crror at the ith location; and $\psi_{i j} \approx\left(\phi_{i}-\phi_{j}\right)$ is the phase difference of the trajectory between the ith and the jth locations phases $\phi_{\mathrm{i}}$ and $\phi_{\mathrm{j}}$.

The optimal procedure has two steps. In the first step a certain linear set of equations has to be solved, and in the second step some matching of the calculated and the measured values has to be performed. The first step can be carried out in either of two ways. Both ways are outlined below under the headings "Procedure 1" and "Alternate Procedure 1."

\section{STEP 1 (PROCEDURE 1)}

Suppose we minimize function I with respect to the phase differences $\psi_{i j}$ thereby considering the $\psi_{i j}$ as variables. Having obtained the equations from variation of $I$ with respect to $\psi_{i j}$, we ask another question: What values should $f_{i}$, (i.c errors) assume, such that the given $\psi_{i j}$ satisfy the variational equations? If we answer this question, then we have found that for the calculated values of $f_{i}$, given $\psi_{i j}$ minimize the amplitude function I. However, it is important to point out the following.

Notice that for $i \neq j, \psi_{i j}=-\psi_{j i}$ and $\psi_{i i}=0$. We are left with only $n(n-1) / 2$ variables, bu' all of these $n(n-1) / 2, \psi_{i j}$ are not linearly independent. Since we are dealing with the phase differences, we can fix a position, say position number ' 1 ', and compute $\psi_{i j}$ for all $j \neq 1$, out of the total of $n(n-1) / 2$, $\psi_{i j}$. Then it is easy to see that for all $i$ and $j, \phi_{i j}=\phi_{1 j}-\phi_{1 i}$. Hence for ' $n$ ' positions corresponding to the ' $n$ ' magnet, there are only $(n-1)$ linearly independent $\psi_{1 j}$. Therefore, to minimize I with respect to the phase differences we will give variations only with respect to $\psi_{1 j}$. Carrying out the variation and assuming that at least one of the errors, say $f_{1}$, is known we get,

$$
\sum_{i=2}^{i=n} H\left(\beta_{i}, L_{i}\right) f_{j}, \sin \psi_{k i}=H\left(\beta_{1}, L_{1}\right) f_{1} \sin \psi_{1 k} \text {, }
$$

which can be formally written as a matrix equation:

$$
\sum_{i=2}^{i=n} M_{k i} f_{i}=H\left(\beta_{1}, L_{1}\right) f_{1} \sin \psi_{1 k} .
$$

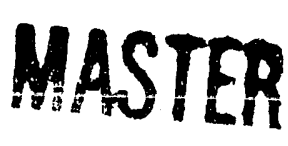

The subenisted manuscribt has treen authored by a contractor of the U.S. Government under contract No. W.31.109ENG.38. Accordingly, the U.S. Government retains a

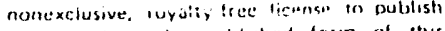
or reproduce the puthished form of this comsribution, or allow others to do sio, for U. S. Governmentl murposes. 


\section{DISCLAIMER}

This report was prepared as an account of work sponsored by an agency of the United States Government. Neither the United States Governinent nor any agency thereof, nor any of their employees, makes any warranty, express or implied, or assumes any legal liability or responsibility for the accuracy, completeness, or usefulness of any information, apparatus, product, or process disclosed, or represents that its use would not infringe privately owned rights. Reference herein to any specific commercial product, process, or service by trade name, trademark, manufacturer, or otherwise does not necessarily constitute or imply its endorsement, recommendation, or favoring by the United States Government or any agency thereof. The views and opinions of authors expressed herein do not necessarily state or reflect those of the United States Government or any agency thereof. 
The Eq. (2) $)^{1,2}$ is true for all $k, k=(2, \ldots, n)$. It is important to remember that the calculated values $\left(f_{2}, \ldots, f_{n}\right)$ will be different from the actual measured values in the magnets. However we will delay the discussion of this point.

\section{STEP 1 (ALTERNATE PROCEDURE 1)}

We assume that at least one of the errors is known and is different from zero. We next consider the rest of the $f_{i}$ as variables and take the derivative of the amplitude function I with respect to these $\int_{i}{ }^{\prime} s$. Lel us assume that we know the value of the first error. Then $\frac{\delta I}{\delta f_{k}}=0$ is a set of linear equations whose solution, in terms of the known phases and the known errors, gives the set of unknown errors whose placement at the corresponding phases would minimize I. Carrying out the above derivative and rearranging the terms, we get for all $k, k=(2, \ldots$, n),

$$
\sum_{j=2}^{j=n} H\left(\beta_{j}, L_{j}\right) f_{j} \cos \psi_{k j}=-H\left(\beta_{1}, L_{1}\right) f_{1} \cos \psi_{k 1} .
$$

Comments similar to ones made in "Procedure 1" are valid for this alternate procedure.

\section{STEP 2}

Having solved the set of linear equations obtained from one of the above procedures, we ask how does this information get translated into the placement of the measured error values. It was answered in the following way. We ordered the measured error values and compared the largest measured error value to the calculated values. The measured error was placed in the position of its closest calculated value. Starting from the largest absolute error value we worked our way through towards the smallest error ${ }^{3}$. In this way we associate the measured errors with a position in phase.

\section{IMPLEMENTATION OF THE OPTIMAL PROCEDURE}

We simulated 45 machines for the dipole errors in dipoles for the APS injector synchrotron. Figure 1 shows the sample random distribution functions used in the simulation. Figures 2 and 3 show the frequency with which the amplification factor I was improved in each machine, over the randomly placed dipoles and quadrupoles, respectively.

\footnotetext{
It may be pointed out that it is not necessary to specify only one of the unknowns to stant. We can specify more than one measured value at different positions and solve for the remaining ones in terms of the known quantities.

${ }^{2}$ Even though we formally have (n-1) linearly independent equations in Eq. (2), it may still be that a set of equations is not linearly independent by virtue of the coefficient of the matrix $M_{k, i}$ vanishing for certain values. ${ }^{3}$ Note that as we go from the largest value to the smaller values the number of choices for measured error to be placed keeps decreasing. In particular, the last measured error has only one place to go. Therefore, this may not be an optimal way to relate measured values to the calculated values.
}
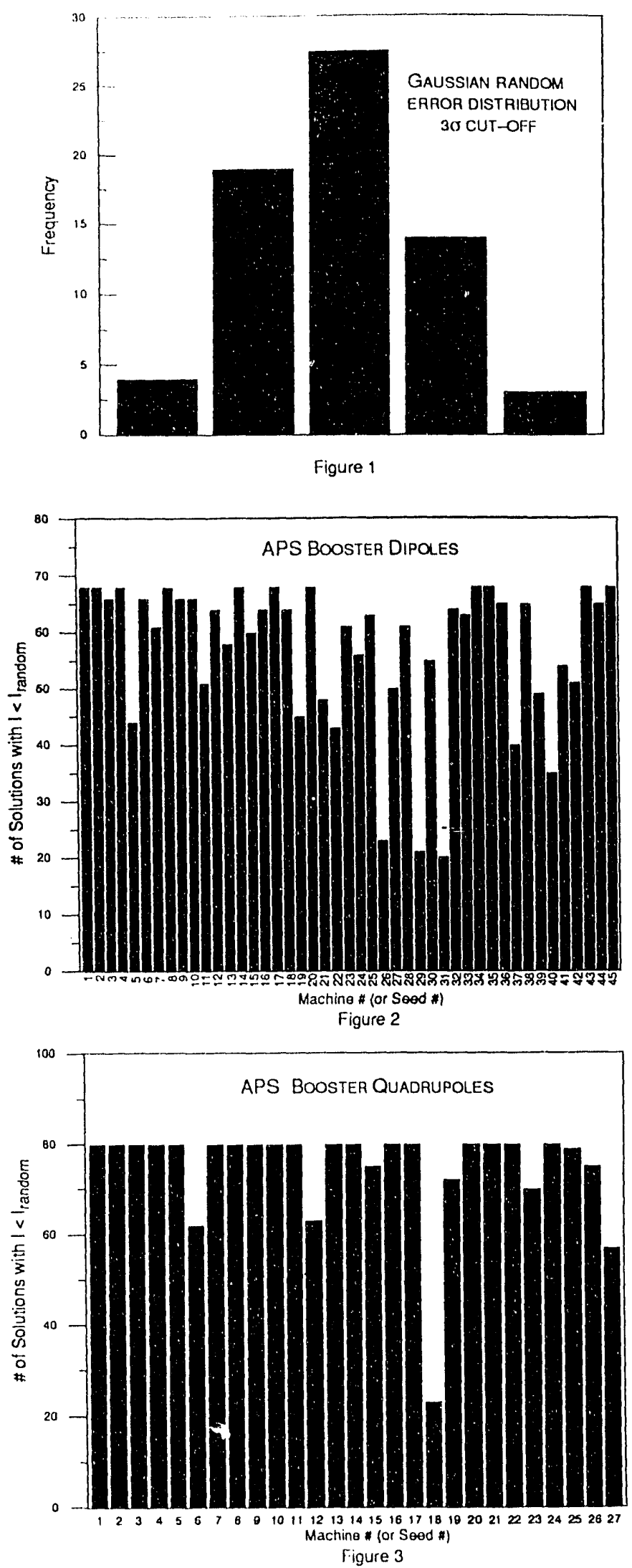

The only comment in favor of this procedure is that it worked extremely well for the simulations nun with simulated random errors used for the APS injector synchrotron dipole and quadrupole errors. 
The frequency distribution of the amplification factor for sorted and random machines is shown in Figure 4. Figure 5 is a similar plot, but here both sets in the plot refer to the amplification factors obtained after sorting. One,corresponds to sorting 68 dipoles at a time and the other corre'sponds to sorting 34 magnets at a time.
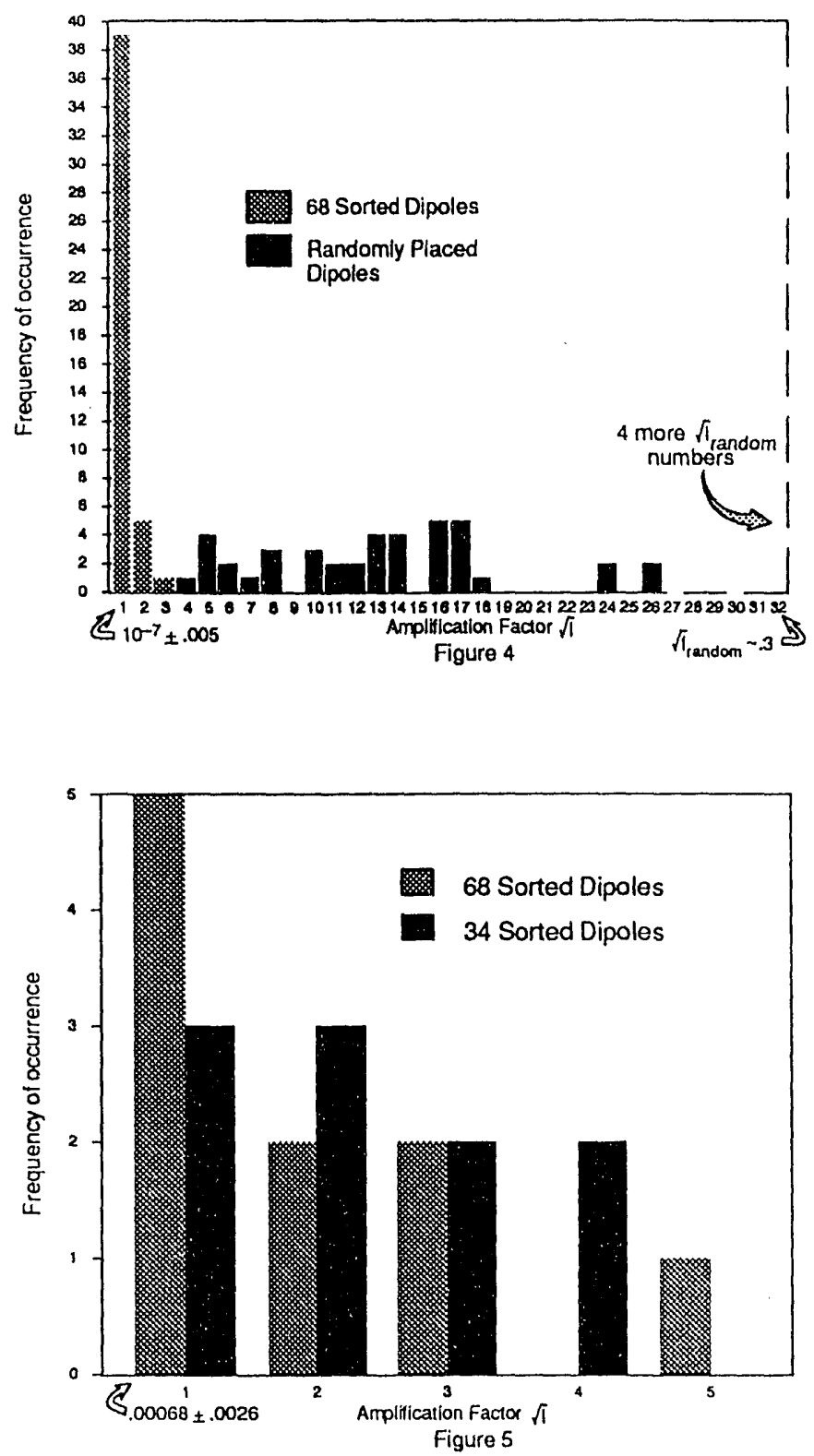

Figure 6 shows the dependence of the result of sorting on the number of magnets sorted at a time. One can easily see that the larger the number of magnets sorted at a time the lower the amplification factor obtained. Figure 6 also shows the mean and the minimum $\sqrt{I_{\text {random. }}}$. One can easily see that not only is the mean $\sqrt{I_{\text {random }}}$ much larger than any of the sorted $\sqrt{I_{\text {racan }}}$, but the minimum $\sqrt{I_{\text {random }}}$ out of 45 random machines is much larger than the sorted $\sqrt{I_{\text {mean }}}$ as long as the number of magnets sorted is at least 18. Figure 7 shows the same result, but here the plot is for the gain factor, defined as $\sqrt{I_{\text {random }} / l_{\text {sorted }}}$, as a function of the number of dipoles sorted.
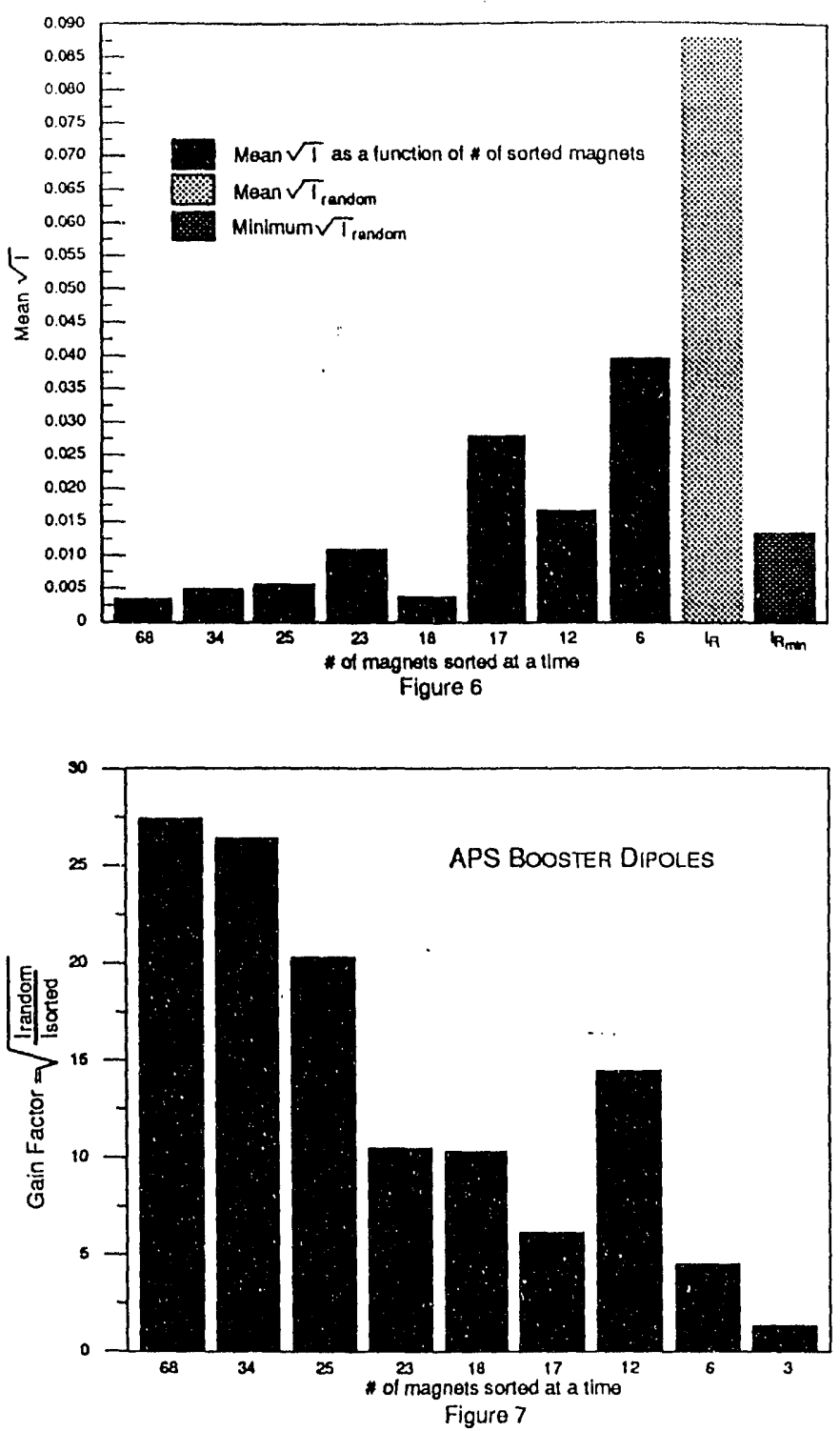

\section{REFERENCES}

[1] Frederick Lopez, private communication.

[2] S. Sampson, "Reduction of Closed Orbit Distortion at the APS Synchrotron by Method of Simulated Annealing," Internal Summer Student Report, APS, Argonne, 1992.

[3] S. Kirkpatrick, "Optimization by Simulated Annealing: Quantitative Studies," Journal of Statistical Physics, Vol. 34, p. $975,1984$.

[4] B. P. Flannery, W. H. Press, S. A. Teukolsky, W. T. Velterling, Numerical Recipes in C The Art Of Scientific Computing, Cambridge University Press, Cambridge, 1989.

[5] B. K. Koul and Frederick Mills, "Linearized Error Analysis for an Accelerator," (to be published).

[6] E. D. Courant and H. S. Snyder, "Theory of Alternating Gradient Synchrotron," Ann. Phys. 3, 1, 1958. 

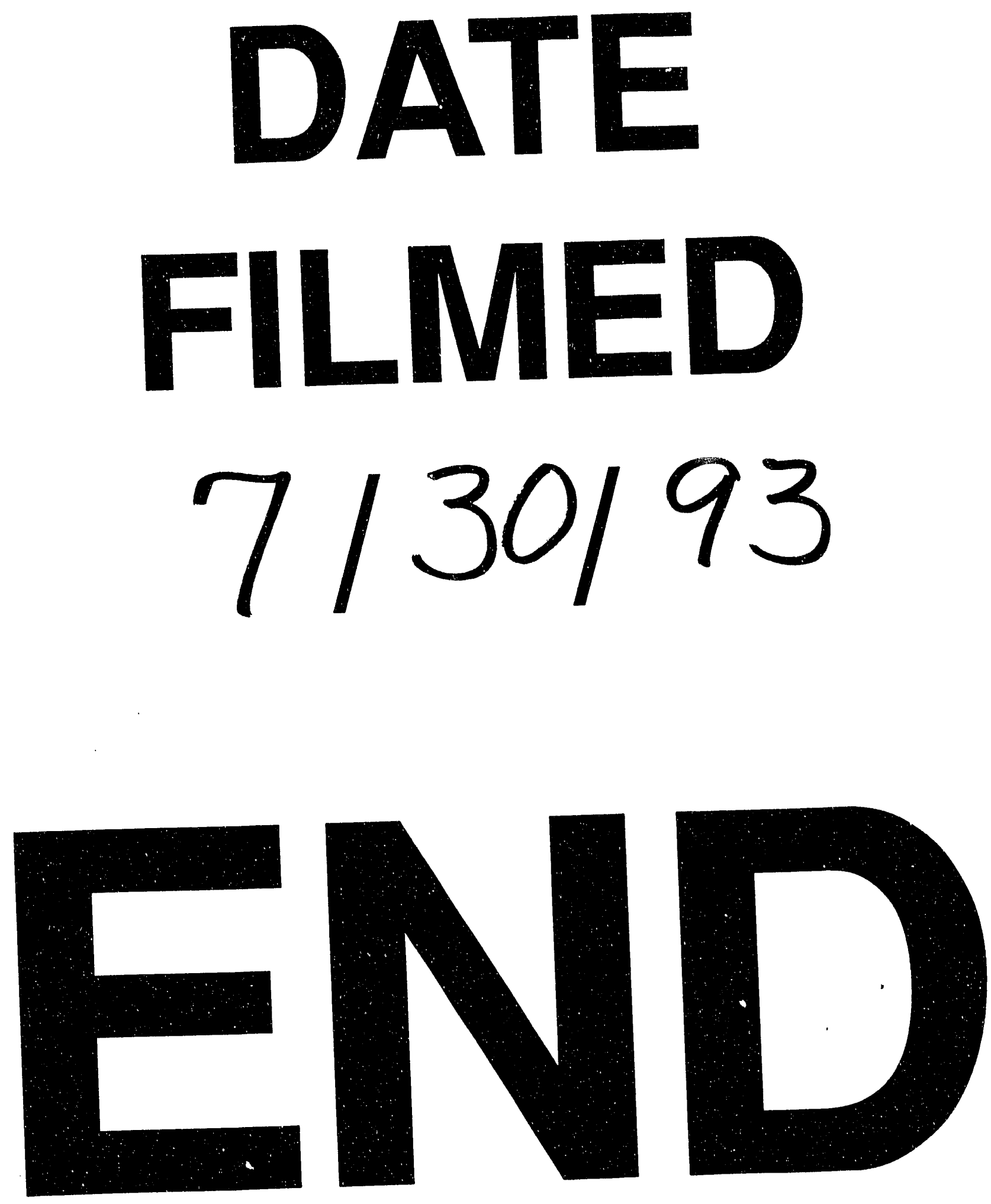
